\title{
Trophic ecology of the ichthyofauna of a stretch of the Urucu River (Coari, Amazonas, Brazil)
}

\author{
Ecologia trófica da ictiofauna de um trecho do rio Urucu (Coari, Amazonas, Brasil)
}

Igor David da Costa ${ }^{1}$ and Carlos Edwar de Carvalho Freitas ${ }^{2}$

\author{
${ }^{1}$ Departamento de Engenharia de Pesca e Aquicultura - DEPA, \\ Fundação Universidade Federal de Rondônia - UNIR, \\ Rua da Paz, 4376, CEP 76916-000, Presidente Médici, RO, Brazil \\ e-mail: igorbiologia@yahoo.com.br \\ ${ }^{2}$ Departmento de Ciências Pesqueiras, Universidade Federal do Amazonas - UFAM, \\ Av. Gen. Rodrigo Otávio, 3000, CEP 69077-000, Manaus, AM, Brazil \\ e-mail: cefreitas@ufam.edu.br
}

\begin{abstract}
Introduction: The floodplains of the large Amazonian rivers are very productive as a result of seasonal fluctuations of water levels. This favors the fishes as they are provided with a wide range of habitats and food resources; Aim: In this study, we identified the trophic structure of fish assemblages in the upper river Urucu area (State of Amazonas - Brazil), observing seasonal changes determined by the hydrological cycle; Methods: Samples were collected with the aid of gillnets, during the flood season (April/2008) and the dry season (August/2008) in areas upstream and downstream of ports of the Urucu river within the municipality of Coari, Amazonas, Brazil; Results: 902 individuals of seven orders, 23 families and 82 species were collected. Fishes were more abundant in the dry season than in the flood season, and the piscivores and carnivores (Serrasalumus rhombeus and Osteoglossum bicirrhosum) were the most significant trophic categories in the dry season whereas piscivores and insectivores (Serrasalumus rhombeus, Bryconops alburnoides and Dianema urostriatum) were more abundant in the flood season. The trophic diversity, dominance and evenness were very similar in all sampling periods and show lower values than taxonomic index patterns, except for the trophic dominance in the dry season. Taxonomic diversity and dominance were higher in the flood season if compared to the dry season, but figures were quite uniform and there were no great discrepancies between seasons. Conclusion: We found through our studies that the dry and flood seasons work as regulatory factors of abundance of fishes of certain trophic categories in the Urucu river, what can be possibly explained by the availability of resources and the food spectrum of each category.
\end{abstract}

Keywords: feeding habits, Amazon, fish, Urucu river, hydrological cycle.

Resumo: Introdução: As áreas inundáveis dos grandes rios amazônicos são muito produtivas, como resultado das flutuaçóes sazonais no nível da água, favorecendo os peixes que passam a dispor de um amplo espectro de habitats e recursos alimentares; Objetivos: Neste estudo, identificamos a estrutura trófica das assembléias de peixes do curso superior do rio Urucu (Amazonas - Brasil), observando mudanças sazonais determinadas pelo ciclo hidrológico; Métodos: As coletas foram realizadas com auxilio de baterias de malhadeiras, no período de cheia (abril/2008) e seca (agosto/2008), em áreas a montante e jusante de portos localizados no rio Urucu, no município de Coari, Amazonas, Brasil; Resultados: Foram coletados 902 indivíduos compreendidos em sete ordens, 23 famílias e 82 espécies, a abundância de peixes no período da seca foi superior ao período da cheia. Piscívoros, onívoros e carnívoros foram as categorias com abundância mais elevada na seca; sendo essas mesmas categorias, incluso o grupo dos detritívoros, mais abundantes na cheia. Bryconops alburnoides, Dianema urostriatum e Serrasalumus rhombeus foram as mais abundantes no período da cheia e Osteoglossum bicirrhosum, Serrasalumus rhombeus e no período da seca; Conclusáo: Constatamos através de nossos estudos a importância dos períodos de seca e cheia como fatores reguladores da abundância de peixes de determinadas categorias tróficas ocorrentes no rio Urucu, que possivelmente podem ser explicadas pela disponibilidade de recursos e espectro alimentar de cada categoria.

Palavras-chave: alimentação, Amazônia, peixes, rio Urucu, ciclo hidrológico. 


\section{Introduction}

The floodplains of the large Amazonian rivers reach an area of about $200,000 \mathrm{~km}^{2}$ and are known locally as várzeas and igapós (Junk, 1993). These areas are very productive and, when flooded, they increase the diversity of habitats that are colonized by several species of fishes. The fluctuation of water levels promotes an alternance of terrestrial and aquatic phases that results in drastic changes in environmental conditions, requiring specific adaptations of the ichthyofauna (Junk et al., 1983). During the falling water and dry seasons, the retraction of water limits the space, forcing fishes to migrate from the floodplain to the main channel of rivers or to permanent lakes (Goulding, 1980). During rising water and flood seasons, the expansion of flooded lands favors the exploration of various habitats and broadens the food spectrum of fishes (Claro-Junior et al., 2004). Abelha et al. (2001) describe that changes on fish diet could be ruled by seasonal spatial changes of the environment, taking into account that different places and seasons provide different abiotic conditions and availability of food. Diet, as well as reproduction and morphology, is one major component of fish ecology, and may be an important descriptor of the fish assemblage structure (Pouilly et al., 2006). The trophic structure in many studies on the ecology of fish is conceptually defined as a grouping of species into functional groups according to their feeding habits and/or icthyo-trophic categories (Gasalla and Soares, 2001). Thus, species with the same trophic position can be grouped into trophic guilds (Lake et al., 2001; Post, 2002), what can be defined as a grouping of species that exploit the same class of environmental resource in a similar way, without considering their taxonomic position (Fauth et al., 1996). From the knowledge of the diet of a fish community and its fish abundance it is possible to identify the different trophic categories, to infer the structure, evaluate the degree of importance of different trophic levels and understand the interrelationships among the components of that community (Agostinho et al., 1997) thus creating a base for a better understanding of the relationships between these components of the ichthyofauna and between these and other organisms of the aquatic community. Knowledge of food sources used by the fishes can provide data on the habitat, food availability in the environment and even on some aspects of behavior (Hahn et al., 1997). Thus, the objective of this work is to characterize the temporal trophic structure of fish species of a stretch of the Urucu River.

\section{Material and Methods}

This study was conducted in a stretch of the Urucu River, within the municipality of Coari, State of Amazonas ( $600 \mathrm{~km}$ west of Manaus, Brazil). The Urucu River $\left(04^{\circ} 53^{\prime} \mathrm{S}\right.$ and $\left.65^{\circ} 11^{\prime} \mathrm{W}\right)$ is a tributary of the right bank of the Solimóes River and one of the main tributaries of Lake Coari (Santos Junior, 2003). The region presents flat or mildly hilly relief, with acid soil (Vieira and Santos, 1987), presents lowland dense ombrophilous forest vegetation with a closed canopy, and is classified as a dense tropical region in the low plateaus sub-region of the Amazon (DNPM, 1978). Since the late 1980s oil and gas have been explored in the Urucu River basin and the sampling points are located near supply ports of oil and gas production facilities. The six sampling sites were located $1 \mathrm{~km}$ upstream and downstream of each port, and were all within a $94.5 \mathrm{~km}$ segment of the river. Fishes were collected during the flood season (April/2008) and dry season (August/2008) in the surroundings of Evandro 1, Evandro 2 and Evandro ports at the following points: control point (PCONT) ( $4^{\circ} 51^{\prime}$ $20,7^{\prime \prime} \mathrm{S}$ and $65^{\circ} 20^{\prime} 53,2^{\prime \prime} \mathrm{W}$ ) located upstream of all ports; a point downstream of Port Urucu (PJU) ( $4^{\circ} 50^{\prime} 59,3^{\prime \prime} \mathrm{S}$ and $65^{\circ} 20^{\prime} 37,4^{\prime \prime} \mathrm{W}$ ); a point just in front of Port Evandro 2 (PPE2) ( $4^{\circ} 45^{\prime} 47,9^{\prime \prime} \mathrm{S}$ and $\left.65^{\circ} 02^{\prime} 46,6^{\prime \prime} \mathrm{W}\right)$; a point upstream of Port Evandro 2 (PME2) (4 $45^{\prime} 42^{\prime \prime} \mathrm{S}$ and $65^{\circ} 20^{\prime} 37,4^{\prime \prime}$ $\mathrm{W})$ and a point downstream of the same port (PJE2) ( $4^{\circ} 45^{\prime} 26,4^{\prime \prime} \mathrm{S}$ and $65^{\circ} 02^{\prime} 38,7^{\prime \prime} \mathrm{W}$ ) and a point downstream of Port Evandro 1 (PJE1) ( $4^{\circ} 45^{\prime}$ $02,2^{\prime \prime} \mathrm{S}$ and $65^{\circ} 02^{\prime} 42,6^{\prime \prime} \mathrm{W}$ ) (Figure 1).

The sampling sites selected for this study were chosen near the docking ports of vessels, owing to the fact that the data for this study came from a larger study that aimed to examine the structure of fish assemblages which revealed, according to Costa (2009), that the assemblages studied in this stretch of river in both periods of the hydrological cycle were not modified as a result of port activities. To minimize the effects of selectivity, gillnets with different mesh sizes were used, organized in groups of eight nets with mesh sizes of 30, 40, 50, 60, 70, 80, 90 e $100 \mathrm{~mm}$ between adjacent knots, each net with a standard size of $20 \mathrm{~m}$ in length and $2 \mathrm{~m}$ height. Overall, gillnets were set out between 06:00 PM and 12:00 AM, aiming to encompass one of the periods of maximum activity for fish (dawn and dusk), and the nets were checked each four hours. The fishes were sorted, identified as according to Nelson (1994), Ferreira et al. (1998) and by experts and fixed in 10\% formalin and 


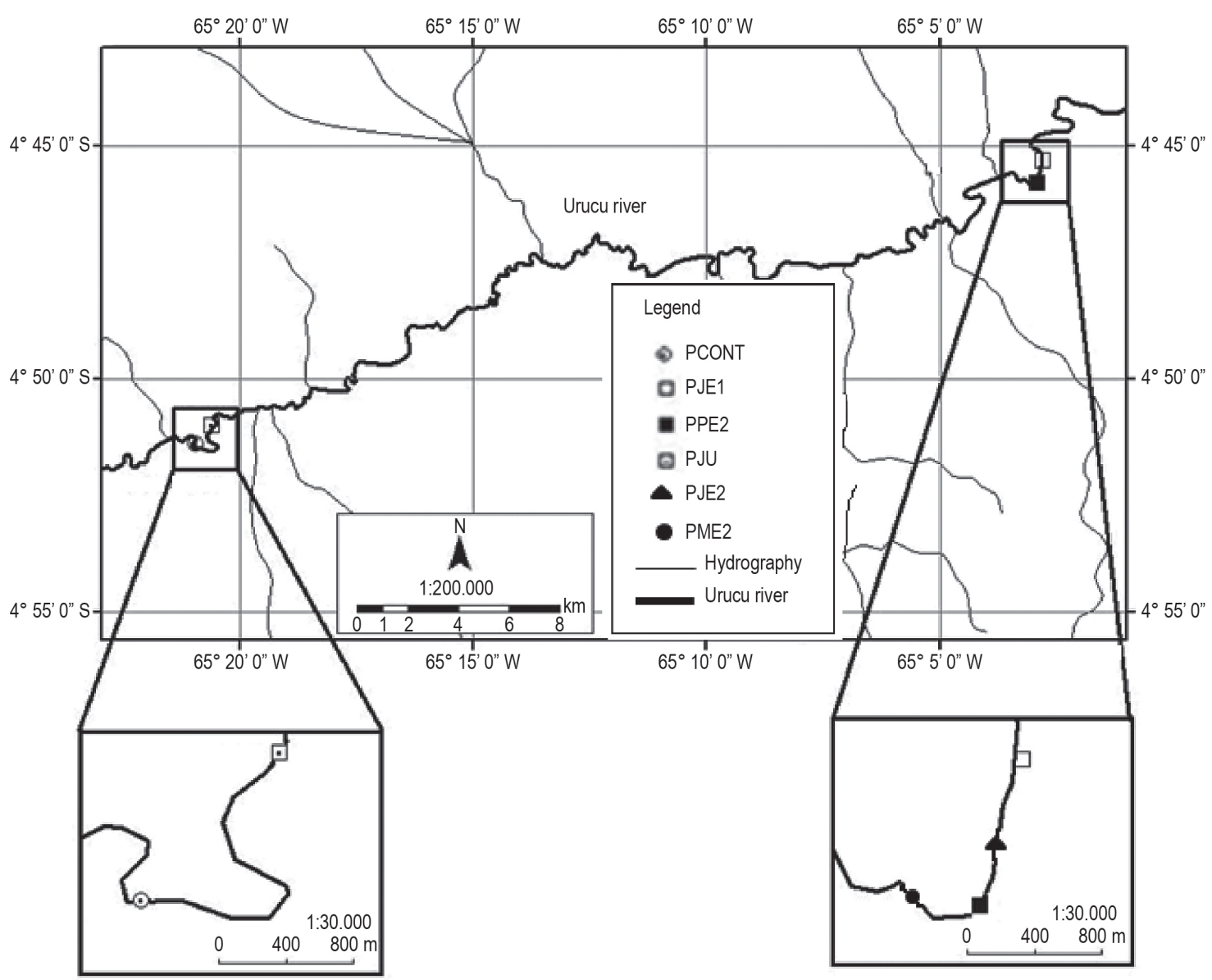

Figure 1. Study area with indication of the sampling points.

preserved in 70\% ethanol, and some specimens were stored in the ichthyologic collection of INPA (asset registration numbers: INPA-32193, INPA-32201, INPA-32186, INPA-32187, INPA-32188, INPA-32184, INPA-32190, INPA-32194, INPA-32195, INPA-32197, INPA-32199, INPA-32183, INPA-32178, INPA-32200, INPA-32175). As an exploratory analysis, we conducted a Nonmetric Multidimensional Scaling (NMDS) with numerical abundance and biomass data of each trophic category according to the seasons (dry vs. flood season) in order to reduce the data dimensionality of the fish assemblage. The dissimilarity matrix used in this ordination was constructed using the Bray-Curtis index. In order to verify the differences between dry and flood periods, we applied the Bifactor Analysis of Similarity (ANOSIM) proposed by Clarke and Green (1988) with data on numerical and biomass abundance. We used the quantitative similarity measure with the Morisita-Horn index (Magurran, 1988), recommended for being almost independent of sample size and species richness (Wolda, 1981). The data was previously $\log$-transformed $(\log x+1)$. To analyze the taxonomic structure of the assemblages in each period, the Alpha diversity was calculated by the Shannon-Wiener index, evenness by the Pielou index (e) (Ludwig and Reynolds, 1988) and to measure the overall species dominance we used the Berger-Parker index (d) (Magurran, 2004). The same indexes mentioned above were also calculated based on the number of trophic categories (richness categories) and number of individuals (abundance) belonging to each category for the sampling periods. Evisceration and analysis of stomach contents were not performed. The trophic categorization of the sampled species was described through the use of bibliographical references, and these were classified and described as detritivores, herbivores (more than 60\% of the diet consists of Phanerogam plant structures); omnivores (when no feature of animal or vegetable origin alone reaches more than $60 \%$ of the diet), insectivores (over $60 \%$ of the diet consists of aquatic and terrestrial insects); invertivores (more than $60 \%$ of the diet is generally comprised of invertebrates, including insects, but 
with no preference for the latter), carnivores (over $60 \%$ of the diet comprises various types of animal resources, such as vertebrates and invertebrates), piscivores (more than $60 \%$ of the diet is composed by fish, fish larvae or fins and scales) (Röpke, 2008), iliophagous (ingest silt or sand substrate looking for food of animal, plant or detritus origins) planktivores (predominantly feed on plankton) (Zavala-Camin, 1996). NMDS and ANOSIM were carried out with the help of the PAST 2.17 software. Inferential analyses were considered significant at $\mathrm{p} \leq 0.05$. In order to better understand the state of those environments, we describe in Table 1 the physical conditions of each sampling point in the Urucu River. Environmental conditions at each sampling point were evaluated according to visual observation of the area; depth $(\mathrm{m})$ with the aid of a measuring tape with a weight; transparency $(\mathrm{cm})$ with a Sechi disk; width (m) of the river with the aid of a GPS; and water flow velocity with a model $2030 \mathrm{R}$ mechanical flow meter that has a 6 digit odometer style counter and can indicate a minimum velocity of $10 \mathrm{~cm} / \mathrm{s}$.

\section{Results}

We collected 902 individuals distributed in seven orders, 23 families and 82 species making up a total biomass of $166.819 \mathrm{~g}$. The species Bryconops alburnoides (Kner, 1858), Dianema urostriatum (Miranda Ribeiro, 1912) and Serrasalumus rhombeus (Linnaeus, 1766) were the most abundant during the flood season and Osteoglossum bicirrhosum
(Cuvier, 1829), Serrasalumus rhombeus (Linnaeus, 1766) in the dry season (Table 2).

The NMDS of samples based on abundance and biomass indicates a separation between the dry and flood seasons on axes 1 and 2, retained for interpretation $\left(\right.$ Stress $_{\text {Abundance }}=0.2 ;$ Stress $\left._{\text {Biomass }=} 0.2\right)$ (Figures 2 and 3).

Analysis of Similarity (ANOSIM) performed for the periods of the hydrological cycle with numerical abundance and biomass data showed no significant difference between the dry and the flood seasons based on biomass $(r=0.15, p=0.10 ; 9999$ permutations). The dry season showed a greater variation in numerical abundance when compared to the flood season, and the two seasons differed significantly in terms of trophic composition $(\mathrm{r}=0.22, \mathrm{p}=0.04,9999$ permutations) (Figure 4), mainly showing a greater abundance of piscivores $(\mathrm{n}=149,34 \%)$ and carnivores $(\mathrm{n}=94,21 \%)$.

A total of eight trophic categories were identified in both seasons. For the dry season the group of piscivores and carnivores were the most significant in abundance $(\mathrm{n}=243 ; 55 \%)$ and biomass $(\mathrm{B}=84.066 \mathrm{~g} ; 78 \%)$. During the flood season these categories were also the ones that showed higher biomass ( $\mathrm{B}=56.897 \mathrm{~g} ; 71 \%)$. Piscivores $(\mathrm{n}=160 ; 34 \%)$ and insectivores $(\mathrm{n}=147 ; 31 \%)$ were the categories with the highest number of individuals during the flood season. Piscivores showed higher abundance $(\mathrm{n}=160,34 \%)$ and biomass ( $\mathrm{B}=38.430 \mathrm{~g} ; 51 \%)$ during the flood season if compared to the dry season $(n=149,33 \%$,

Table 1. Description of the conditions of each sampling point in the Urucu River, Coari municipality - AM/Brazil.

Points

PCONT Very steep river banks, narrow river, absence of rapids and macrophytes, river containing fairly closed canopy trees, absence of "igapós", local banks preserved (no fallen trees owing to the action of rafts or ferries), absence of ferry traffic, absence oil in the water. Transparency $=65 \mathrm{~cm}$, water flow velocity $=0.33 \pm 0.50 \mathrm{~m} / \mathrm{s}$, river width $=14 \mathrm{~m}$ and depth $=1.15 \mathrm{~m}$.

PJU Steep river banks with small portions of "igapós", absence of rapids and macrophytes, local banks showing fallen trees owing to the action of rafts or ferries, occurrence of ferry traffic, absence of oil in water; transparency $=53 \mathrm{~cm}$; water flow velocity $=0.33 \pm 0.50 \mathrm{~m} / \mathrm{s}$; river width $=26.3 \mathrm{~m}$; river depth $=1.20 \mathrm{~m}$.

PME2 Steep river banks, "igapós" present, absence of rapids and macrophytes, local banks preserved, occurrence of ferry traffic, absence of oil in water; transparency $=54 \mathrm{~cm}$, water flow velocity $=0.25 \pm 0.33 \mathrm{~m} / \mathrm{s}$, river width $=35 \mathrm{~m}$; river depth $=4.32 \mathrm{~m}$.

PPE2

Wider river, presence of "igapó", absence of rapids and macrophytes, sampling point located next to the port, presence of docked ferries, local river banks preserved, moderate occurrence of ferry traffic, absence of oil in water, transparency $=54 \mathrm{~cm}$, water flow velocity $=0.33 \pm 0.50 \mathrm{~m} / \mathrm{s}$; river width $=44 \mathrm{~m}$; river depth $=1.80 \mathrm{~m}$.

PJE2 Steep river banks, "igapó" presence, absence of rapids and macrophytes, local banks preserved, moderate occurrence of ferry traffic, absence of oil in water, transparency $=72 \mathrm{~cm}$, water flow velocity $=0.33 \pm 0.50 \mathrm{~m} / \mathrm{s}$; river width $=40 \mathrm{~m}$; depth $=7.78 \mathrm{~m}$ transparency $=84 \mathrm{~cm}$; water flow velocity $=020 \pm 0.33 \mathrm{~m} / \mathrm{s}$; river width $=38 \mathrm{~m}$; river depth $=2.92 \mathrm{~m}$ 
Table 2. Data of numerical abundance (A) during flood (dry) periods, trophic categorization (TC) (CAR=Carnivores, DET=Detritivores, HER=Herbivores, ILI=Iliophagous, INS=Insectivores, INV=Invertivores, ONI=omnivores, PIS=piscivores, PLA=planktivores) and references of the species collected in the Urucu River, municipality of Coari - AM / Brazil.

\begin{tabular}{|c|c|c|c|}
\hline Order, Family, Genus and Species & $\mathbf{A}$ & TC & References* \\
\hline \multicolumn{4}{|l|}{ Order Osteoglossiformes } \\
\hline \multicolumn{4}{|l|}{ Family Osteoglossidae } \\
\hline Osteoglossum bicirrhosum (Cuvier, 1829) & $0(55)$ & CAR & Mérona and Rankin-de-Mérona (2004) \\
\hline \multicolumn{4}{|l|}{ Order Clupeiformes } \\
\hline \multicolumn{4}{|l|}{ Family Pristigasteridae } \\
\hline Pellona castelnaeana Valenciennes, 1847 & $32(0)$ & PIS & Mérona and Rankin-de-Mérona (2004) \\
\hline Pellona flavipinnis (Valenciennes, 1837) & $22(4)$ & CAR & Mérona and Rankin-de-Mérona (2004) \\
\hline \multicolumn{4}{|l|}{ Family Engraulidade } \\
\hline Lycengraulis batesii (Günther, 1868) & $7(1)$ & PIS & $\begin{array}{l}\text { Mérona and Rankin-de-Mérona (2004) } \\
\text { (Lycengraulis grossidens) }\end{array}$ \\
\hline \multicolumn{4}{|l|}{ Order Beloniformes } \\
\hline \multicolumn{4}{|l|}{ Family Belonidae } \\
\hline Potamorrhaphis guianensis (Jardine, 1843) & $1(0)$ & CAR & Reis et al. (2003) \\
\hline \multicolumn{4}{|l|}{ Order Characiformes } \\
\hline \multicolumn{4}{|l|}{ Family Acestrorhynchidae } \\
\hline Acestrorhynchus falcirostris (Cuvier, 1819) & $1(28)$ & PIS & Santos et al. (2004) \\
\hline Acestrorhynchus falcatus (Bloch, 1794) & $3(0)$ & PIS & Reis et al. (2003) \\
\hline Acestrorhynchus microlepis (Schomburgk, 1841) & $1(0)$ & PIS & Pouilly et al. (2003) \\
\hline \multicolumn{4}{|l|}{ Family Agoniatidae } \\
\hline Agoniates halecinus Müller \& Troschel, 1845 & $11(14)$ & PIS & Goulding et al. (1988) \\
\hline \multicolumn{4}{|l|}{ Family Anostomidae } \\
\hline Abramites hypselonotus (Günther, 1868) & $0(2)$ & ONI & Santos and Rosa (1998) \\
\hline Leporinus agassizii Steindachner, 1876 & $14(16)$ & ONI & Santos and Rosa (1998) \\
\hline Leporinus fasciatus (Bloch, 1794) & $0(2)$ & ONI & Santos et al. (2006) \\
\hline Schizodon fasciatus Spix \& Agassiz, 1829 & $0(1)$ & HER & Santos (1981) \\
\hline Rhytiodus argenteofuscus Kner, 1858 & $3(0)$ & HER & Santos ( 1981) \\
\hline \multicolumn{4}{|l|}{ Family Characidae } \\
\hline Brycon cf. pesu Müller Troschel, 1845 & $16(6)$ & ONI & Arrington (2002) \\
\hline Brycon melanopterus (Cope, 1872) & $0(4)$ & ONI & Mérona and Rankin-de-Mérona (2004) \\
\hline Bryconops alburnoides Kner, 1858 & $64(5)$ & INS & Silva (2008) \\
\hline Bryconops caudomaculatus (Günther, 1864) & $0(1)$ & INS & Silva (2008) \\
\hline Chalceus erythrurus (Cope, 1870) & 2(19) & INS & Arrington (2002) \\
\hline Moenkhausia lepidura (Kner, 1858) & $14(5)$ & INS & Moura et al. (2010) \\
\hline Myloplus rubripinnis (Müller \& Troschel, 1844) & $4(5)$ & HER & Reis et al. (2003) \\
\hline Myleus sp. & $1(0)$ & HER & Reis et al. (2003) \\
\hline Myleus schomburgkii (Jardine, 1841) & $1(0)$ & HER & Reis et al. (2003) \\
\hline Poptella brevispina Reis, 1989 & $1(0)$ & ONI & $\begin{array}{l}\text { Santos et al. (2009) } \\
\text { (Poptella paraguayensis) }\end{array}$ \\
\hline Pristobrycon striolatus (Steindachner, 1908) & $6(0)$ & PIS & Nico and Tarphon (1988) \\
\hline Pygocentrus nattereri Kner, 1858 & $12(5)$ & PIS & Mérona and Rankin-de-Mérona (2004) \\
\hline Serrasalmus sp. & $0(2)$ & PIS & Reis et al. (2003) \\
\hline Serrasalmus altispinis Merckx, Jégu \& Santos, 2000 & $0(6)$ & PIS & Reis et al. (2003) \\
\hline Serrasalmus rhombeus (Linnaeus, 1766) & $54(44)$ & PIS & Santos et al. (2004) \\
\hline Tetragonopterus chalceus Spix \& Agassiz, 1829 & $1(0)$ & ONI & Ferreira and Santos (2006) \\
\hline Triportheus albus Cope, 1872 & $0(6)$ & ONI & Pouilly et al. (2003) \\
\hline Triportheus angulatus (Spix \& Agassiz, 1829) & $0(7)$ & ONI & Yamamoto et al. (2004) \\
\hline Triportheus elongatus (Günther, 1864) & $1(0)$ & ONI & Mérona and Rankin-de-Mérona (2004) \\
\hline \multicolumn{4}{|l|}{ Family Chilodontidae } \\
\hline Caenotropus labyrinthicus (Kner, 1858) & 2(9) & ONI & Godoi (2004) \\
\hline
\end{tabular}

${ }^{*}$ Arrington (2002), Claro-Junior et al. (2004), Correa (2005), Duarte (2008), Ferraris Junior (1991), Ferreira and Santos (2006), Godoi (2004), Goulding et al. (1988), Gurgel et al. (2002), Marinelli et al. (1993), Mérona and Rankin-de-Mérona (2004), Nico and Tarphon, 1988), Pereira et al. (1996), Moura et al. (2010), Pouilly et al. (2003), Reis (1997), Reis et al. (2003), Rezende et al. (2005), Röpke (com. pess), Santos (1981, 1991), Santos and Rosa (1998), Santos et al. (2004, 2006, 2009), Silva (2008) and Yamamoto et al. (2004). 
Table 2. Continued...

\begin{tabular}{|c|c|c|c|}
\hline Order, Family, Genus and Species & $\mathbf{A}$ & TC & References* \\
\hline \multicolumn{4}{|l|}{ Family Ctenoluciidae } \\
\hline Boulengerella cuvieri (Spix \& Agassiz, 1829) & $2(0)$ & PIS & Duarte (2008) \\
\hline Boulengerella maculata (Valenciennes, 1850) & $3(22)$ & PIS & Goulding et al. (1988) \\
\hline \multicolumn{4}{|l|}{ Family Curimatidae } \\
\hline Curimata cf. cisandina (Allen, 1942) & $1(0)$ & DET & Santos (1991) (C. inornata) \\
\hline Curimata inornata Vari, 1989 & $0(33)$ & DET & Santos (1991) \\
\hline Curimata vittata (Kner, 1858) & $1(3)$ & DET & Santos (1991) \\
\hline Cyphocharax notatus (Steindachner, 1908) & $0(2)$ & DET & Santos et al. (2004) \\
\hline Psectrogaster amazonica Eigenmann \& Eigenmann, 1889 & $1(0)$ & DET & Mérona and Rankin-de-Mérona (2004) \\
\hline Psectrogaster rutiloides (Kner, 1858) & $4(0)$ & DET & Santos (1991) \\
\hline Steindachnerina bimaculata (Steindachner, 1876) & $0(1)$ & ILI & Godoi (2004) \\
\hline \multicolumn{4}{|l|}{ Family Cynodontidae } \\
\hline Cynodon gibbus (Agassiz, 1829) & $3(0)$ & PIS & Santos et al. (2006) \\
\hline Rhaphiodon vulpinus Agassiz, 1829 & $5(0)$ & PIS & Pouilly et al. (2003) \\
\hline \multicolumn{4}{|l|}{ Family Erytrinidae } \\
\hline Hoplias malabaricus (Bloch, 1794) & $1(9)$ & PIS & Mérona and Rankin-de-Mérona (2004) \\
\hline \multicolumn{4}{|l|}{ Family Hemiodontidae } \\
\hline Hemiodus semitaeniatus Kner, 1858 & $2(6)$ & HER & Godoi (2004) \\
\hline Hemiodus unimaculatus (Bloch, 1794) & $7(36)$ & HER & Godoi (2004) \\
\hline Anodus orinocensis (Steindachner, 1887) & $1(0)$ & PLA & Santos et al. (2004) \\
\hline \multicolumn{4}{|l|}{ Order Siluriformes } \\
\hline \multicolumn{4}{|l|}{ Family Loricariidae } \\
\hline Ancistrus sp. & $0(1)$ & DET & Pereira et al. (1996) \\
\hline Dekeyseria amazonica Rapp Py-Daniel, 1985 & $1(0)$ & HER & Rezende et al. (2005) \\
\hline Loricaria cataphracta Linnaeus, 1758 & $0(1)$ & DET & Pereira et al. (1996) \\
\hline Rineloricaria sp. & $0(4)$ & DET & $\begin{array}{l}\text { Pereira et al. (1996) } \\
\text { (Rineloricaria nigricauda) }\end{array}$ \\
\hline Sturisoma sp. & $0(2)$ & DET & $\begin{array}{l}\text { Pereira et al. (1996) } \\
\text { (Sturisoma robustum) }\end{array}$ \\
\hline Hypoptopoma cf. gulare Cope, 1878 & $24(1)$ & ILI & Santos et al. (2004) \\
\hline \multicolumn{4}{|l|}{ Family Auchenipteridae } \\
\hline Ageneiosus inermis (Linnaeus, 1766) & $1(0)$ & CAR & Santos et al. (2006) \\
\hline Ageneiosus gr. vittatus (Steindachner, 1908) & $1(0)$ & CAR & Santos et al. (2006) \\
\hline Auchenipterichthys sp. & $2(0)$ & ONI & $\begin{array}{l}\text { Marinelli et al. (1993) } \\
\text { (Auchenipterichthys thoracatus) }\end{array}$ \\
\hline Auchenipterus ambyiacus Fowler, 1915 & $2(0)$ & CAR & Pouilly et al. (2003) \\
\hline Auchenipterus nuchalis (Spix \& Agassiz, 1829) & $2(0)$ & INS & Mérona and Rankin-de-Mérona (2004) \\
\hline Centromochus heckelli (De Felippi, 1853) & $2(0)$ & CAR & Röpke (com. pess) \\
\hline Trachelyopterus galeatus (Linnaeus, 1766) & $5(0)$ & ONI & Claro-Junior et al. (2004) \\
\hline Tatia intermedia (Steindachner, 1877) & $2(0)$ & INS & Ferraris Junior (1991) \\
\hline \multicolumn{4}{|l|}{ Family Callichthyidae } \\
\hline Dianema urostriatum (Miranda Ribeiro, 1912) & $63(0)$ & INS & $\begin{array}{l}\text { Correa (2005) } \\
\text { (D. longibarbis) }\end{array}$ \\
\hline \multicolumn{4}{|l|}{ Family Pimelodidae } \\
\hline Calophysus macropterus (Lichtenstein, 1819) & $28(15)$ & CAR & Santos et al. (2004) \\
\hline Hemisorubim platyrhynchos (Valenciennes, 1840) & $0(8)$ & CAR & Duarte (2008) \\
\hline Hypophthalmus edentatus Spix \& Agassiz, 1829 & $2(0)$ & PLA & Santos et al. (2006) \\
\hline Hypophthalmus marginatus Valenciennes, 1840 & $2(0)$ & PLA & Santos et al. (2006) \\
\hline Pimelodus blochii Valenciennes, 1840 & $5(4)$ & CAR & Mérona and Rankin-de-Mérona (2004) \\
\hline Pinirampus pirinampu (Spix \& Agassiz, 1829) & $4(0)$ & PIS & Rezende et al. (2005) \\
\hline Pseudoplatystoma fasciatum (Linnaeus, 1766) & $1(0)$ & PIS & Lowe-McConnel (1975) \\
\hline Sorubim lima (Bloch \& Schneider, 1801) & $4(0)$ & CAR & Santos et al. (2006) \\
\hline
\end{tabular}

*Arrington (2002), Claro-Junior et al. (2004), Correa (2005), Duarte (2008), Ferraris Junior (1991), Ferreira and Santos (2006), Godoi (2004), Goulding et al. (1988), Gurgel et al. (2002), Marinelli et al. (1993), Mérona and Rankin-de-Mérona (2004), Nico and Tarphon, 1988), Pereira et al. (1996), Moura et al. (2010), Pouilly et al. (2003), Reis (1997), Reis et al. (2003), Rezende et al. (2005), Röpke (com. pess), Santos (1981, 1991), Santos and Rosa (1998), Santos et al. (2004, 2006, 2009), Silva (2008) and Yamamoto et al. (2004). 
Table 2. Continued...

\begin{tabular}{|c|c|c|c|}
\hline Order, Family, Genus and Species & A & TC & References* \\
\hline \multicolumn{4}{|l|}{ Order Perciformes } \\
\hline \multicolumn{4}{|l|}{ Family Cichlidae } \\
\hline Biotodoma cupido (Heckel, 1840) & $0(6)$ & CAR & Reis (1997) \\
\hline Chaetobranchus flavescens Heckel, 1840 & $0(7)$ & INV & Mérona and Rankin-de-Mérona (2004) \\
\hline Cichla sp. & $0(10)$ & PIS & Mérona and Rankin-de-Mérona (2004) \\
\hline Cichla monoculus Spix \& Agassiz, 1831 & $0(2)$ & PIS & Mérona and Rankin-de-Mérona (2004) \\
\hline Crenicichla cincta Regan, 1905 & $1(0)$ & PIS & $\begin{array}{l}\text { Gurgel et al. (2002) } \\
\text { (Crenicichla menezesi) }\end{array}$ \\
\hline Geophagus proximus (Castelnau, 1855) & $0(9)$ & ONI & Santos et al. (2006) \\
\hline Satanoperca jurupari (Heckel, 1840) & $0(1)$ & ONI & Santos et al. (2004) \\
\hline \multicolumn{4}{|l|}{ Family Scianidae } \\
\hline Plagioscion squamosissimus (Heckel, 1840) & $8(6)$ & CAR & Santos et al. (2004) \\
\hline \multicolumn{4}{|l|}{ Order Pleuronectiformes } \\
\hline \multicolumn{4}{|l|}{ Family Achiridae } \\
\hline Hypoclinemus mentalis (Günther, 1862) & $0(1)$ & CAR & Reis et al. (2003) \\
\hline
\end{tabular}

*Arrington (2002), Claro-Junior et al. (2004), Correa (2005), Duarte (2008), Ferraris Junior (1991), Ferreira and Santos (2006), Godoi (2004), Goulding et al. (1988), Gurgel et al. (2002), Marinelli et al. (1993), Mérona and Rankin-de-Mérona (2004), Nico and Tarphon, 1988), Pereira et al. (1996), Moura et al. (2010), Pouilly et al. (2003), Reis (1997), Reis et al. (2003), Rezende et al. (2005), Röpke (com. pess), Santos (1981, 1991), Santos and Rosa (1998), Santos et al. (2004, 2006, 2009), Silva (2008) and Yamamoto et al. (2004).

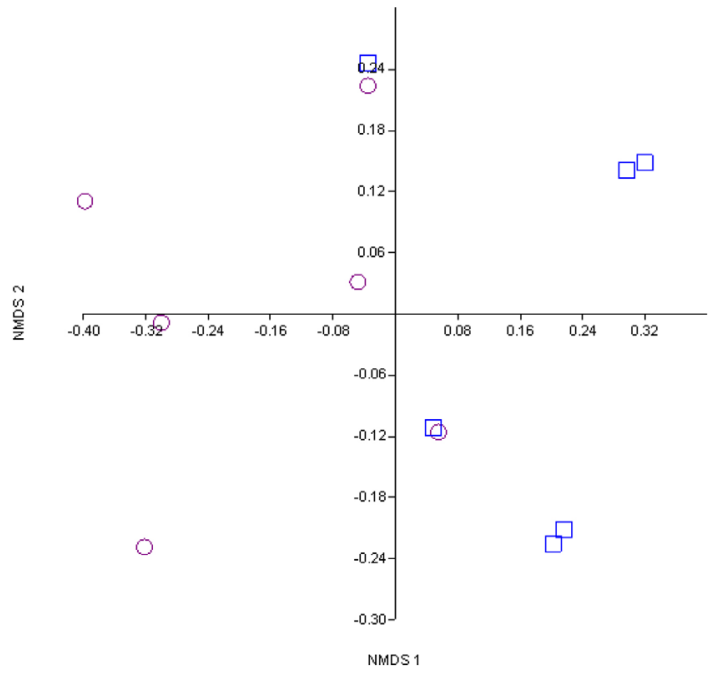

Figure 2. NMDS graphics for the sampling periods based on abundance of trophic categories. $\square=$ flood season and $\mathrm{O}=$ dry season.

$\mathrm{B}=23,138 ; 22 \%$ ), and the group of carnivores were the most significant in terms of biomass in the dry season ( $\mathrm{B}=60.928 \mathrm{~g} ; 57 \%$ ) (Table 3).

Seasonal analysis showed that the taxonomic diversity and dominance were higher in the flood season if compared to dry season, and evenness showed no significant difference between seasons. The trophic diversity, dominance and evenness (trophic indexes) were very similar in the dry and flood seasons, but were lower than taxonomic levels, except for the trophic dominance in the dry season (Table 4).

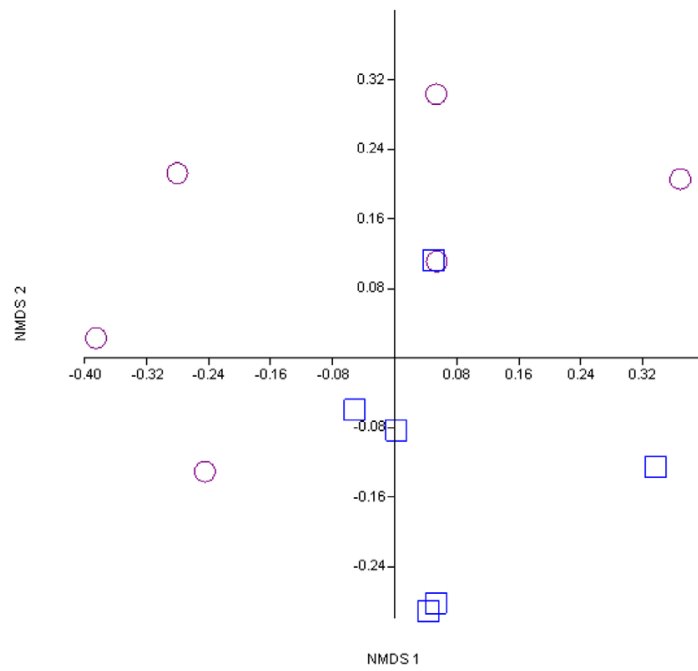

Figure 3. NMDS graph for the sampling periods based on biomass of trophic categories. $\square=$ flood season and $\mathrm{O}=$ dry season.

\section{Discussion}

The predominance of the Otophysi group fishes found in our research is consistent with what was expected for the Amazon region (Nelson, 1994), as it is characterized by the dominance of Characiformes (43\%), Siluriformes (36\%) and Gymnotiformes (3\%) representing approximately $82 \%$ of the Amazon fish fauna (Lowe-McConnell, 1999). This dominance is well reported for various rivers in the Neotropical region (Robertson, 1972; Sabino and Zuanon, 1998; Lowe-McConnell, 1999; Castro, 1999; 


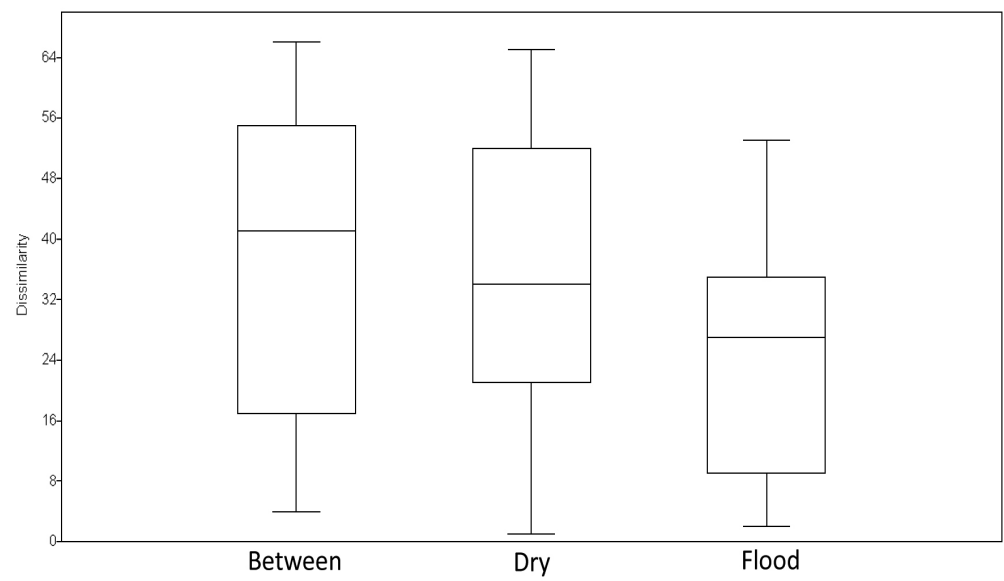

Figure 4. Dissimilarity of abundance of trophic groups in the dry and flood seasons.

Table 3. Data of numerical abundance $(\mathrm{N})$, abundance percentage $(\% \mathrm{~N})$, biomass $(\mathrm{B})$ and biomass percentage $(\% \mathrm{~B})$ for each trophic rank (TR) (CAR=Carnivores, DET=Detritivores, HER=Herbivores, ILI=Iliophagous, INS=Insectivores, $\mathrm{INV}=$ Invertivores, $\mathrm{ONI}=$ omnivores, $\mathrm{PIS}=$ piscivores, $\mathrm{PLA}=$ planktivores) during dry and flood seasons in the Urucu River, municipality of Coari - AM / Brazil.

\begin{tabular}{|c|c|c|c|c|c|c|c|c|c|c|c|}
\hline \multicolumn{6}{|c|}{ Dry } & \multicolumn{6}{|c|}{ Flood } \\
\hline \multicolumn{3}{|c|}{ Abundance } & \multicolumn{3}{|c|}{ Biomass (g) } & \multicolumn{3}{|c|}{ Abundance } & \multicolumn{3}{|c|}{ Biomass (g) } \\
\hline TR & $\mathbf{N}$ & $\% \mathrm{~N}$ & TR & B & $\% \mathrm{~B}$ & TR & $\mathbf{N}$ & $\% \mathrm{~N}$ & TR & B & $\% B$ \\
\hline PIS & 149 & 33.9 & CAR & 60.928 & 57.4 & PIS & 160 & 33.9 & PIS & 38.430 & 50.9 \\
\hline CAR & 94 & 21.4 & PIS & 23.138 & 21.8 & INS & 147 & 31.1 & CAR & 18.467 & 24.5 \\
\hline ONI & 62 & 14.1 & $\mathrm{ONI}$ & 11.288 & 10.6 & CAR & 76 & 16.1 & $\mathrm{ONI}$ & 10.665 & 14.1 \\
\hline HER & 54 & 12.3 & HER & 4.815 & 4.5 & ONI & 46 & 9.75 & HER & 3.78 & 5 \\
\hline DET & 46 & 10.5 & DET & 2.685 & 2.5 & ILI & 24 & 5.08 & INS & 1.77 & 2.3 \\
\hline INS & 25 & 5.7 & INS & 1.595 & 1.5 & HER & 12 & 2.54 & PLA & 873 & 1.2 \\
\hline INV & 7 & 1.6 & INV & 1.58 & 1.5 & DET & 4 & 0.85 & DET & 590 & 0.8 \\
\hline ILI & 2 & 0.5 & ILI & 115 & 0.1 & PLA & 3 & 0.636 & ILI & 475 & 0.6 \\
\hline
\end{tabular}

Table 4. Taxonomic diversity index $\left(\mathrm{H}_{\mathrm{Tax}}{ }_{\mathrm{Tax}}\right)$, taxonomic dominance index $\left(\mathrm{d}_{\text {Tax }}\right)$, Pielou's taxonomic evenness index $\left(\mathrm{e}_{\mathrm{Tax}}\right)$, trophic diversity index $\left(\mathrm{H}^{\prime}{ }_{\mathrm{Trof}}\right)$, trophic dominance index $\left(\mathrm{d}_{\text {Trof }}\right)$ and Pielou's trophic evenness index $\left(\mathrm{e}_{\text {Trof }}\right)$ for the six sampled stretches of the Urucu River during the dry and flood seasons.

\begin{tabular}{ccccccc}
\hline Season & $\mathbf{H}_{\text {Tax }}^{\prime}$ & $\mathbf{H}_{\text {Trof }}^{\prime}$ & $\mathbf{d}_{\text {Tax }}$ & $\mathbf{d}_{\text {Trof }}$ & $\mathbf{e}_{\text {Tax }}$ & $\mathbf{e}_{\text {Trof }}$ \\
\hline Dry & 4.70 & 2.40 & 0.20 & 0.32 & 0.83 & 0.80 \\
Flood & 4.80 & 2.43 & 0.60 & 0.30 & 0.81 & 0.80 \\
\hline
\end{tabular}

Pouilly et al., 2004). The most abundant families in the sampled stretches and seasons were Characidae and Loricariidae, groups widely distributed throughout the Amazon basin (Britski, 1999; Ferreira et al., 1998; Santos et al., 2006).

An important factor for the structuring of fish communities in the tropics is related to the mobility of species, since many of these migrate from rivers into and out of lakes, flooded forests and tributaries according to the changes of water levels and physical/ chemical variables (Goulding, 1980). In the flood plain areas, the diversity and density of fishes are determined by the flow regime; flooding increases homogenization of limnological characteristics of the environment (Thomaz et al., 1997) while dry season makes the environments respond to regional factors independently, increasing fauna heterogeneity as microhabitats become isolated (Teixeira et al., 2005). Our exploratory analysis identified a segregation between dry and flood seasons based on the abundance and biomass of trophic categories, and greater variability between sites in the dry season (see Figure 4). Thus, we infer that the contribution of each trophic group, for each sample season, acts in a distinct way in the Urucu River. Such condition corroborates scientific literature. For example, based only on information about piscivore guilds, studies indicate that this category is the most significant in abundance and biomass during dry season because there is a higher density of prey in the river channels (Saint-Paul et al., 2000; Claro-Junior, 2003; Júlio Junior et al., 2003; Siqueira-Souza and Freitas, 2004; Doria et al., 2005; Correa, 2008; Sousa and Freitas, 2008). As well as to the flood season, where Pompeu (1997) describes that river bank areas are 
flooded supplying a greater amount of food and prey, which in turn supports higher densities of fish. Röpke (2008), in a study of temporal variation of trophic structure in aquatic herbaceous biotopes, points to a higher number of individuals and high piscivore biomass in dry and receding waters periods. Our results showed high contribution of carnivore and piscivore species (e.g. O. bicirrhosum, $S$. rhombeus) in both periods, and insectivores (e.g. D. urostriatum e B. alburnoides) in the flood period. The flood season offers a wide range of food items, favoring insectivore and omnivore relations, whereas the dry season favors carnivore relations (Santos et al., 2009) owing to the increase of fish density, what intensifies the biotic interactions in tropical aquatic ecosystems, such as predation (Layman and Winemiller, 2004).

In general, abundance levels are lower in the flood season owing to the dispersion of fish in the environment, making it difficult to capture them with fishing equipment. In addition, in the dry season fish are more concentrated owing to the reduction of the water body area, what makes fish catching easier (Lowe-McConell, 1999). However, in contradiction with literature, greater absolute abundance and biomass of piscivores were sampled during flood season when compared to dry season. According to Jepsen (1997), in studies on the Cinaruco river (Venezuela), during the dry season larger prey $(>110 \mathrm{~mm})$ are less abundant apparently due to intense predation during the period of flood and receding waters, confirming our results in that relates to the high number of piscivores in the flood season.

The stability of the environment is an important condition for predator species of specialized feeding habits while there is a greater number of species that have broader feeding habits in environments that are more variable, which are subject to disturbances and to a rich range of piscivore species in stable environmental conditions (Kushlan, 1976; Poff and Allan, 1995; Hoeinghaus et al., 2006), as in the environment we studied. The occurrence of a high level of piscivory in certain tropical systems has been attributed to the abundance of detritivore habit prey in floodplains (Winemiller and Jepsen, 1998; Winemiller, 2004). Seasonal changes in the composition of the ichthyofauna were also observed in the floodplains of the Rupunini River (Lowe-Mcconnell, 1975), the Machado and Madeira rivers (Goulding, 1980), Lake Inácio (Saint-Paul et al., 2000), Lago do Rei (Mérona and Bittencourt, 1993), Catalão Lake (Vale, 2003) and
Lakes Samaúma, Sacambú and Maracá in Central Amazon (Siqueira-Souza and Freitas, 2004).

In the studied area the diversity values obtained can be considered high, considering that index values above 3.0 indicate high diversity (Goulding, 1980). Corroborating our results, Kushlan (1976), Saint-Paul et al. (2000), Ferreira and Casatti (2006), Cunico et al. (2006), Ferreira and Petrere (2007), Silva (2008) and Correa (2008) found higher values of taxonomic diversity in the flood season. The dynamics of flooding promotes increased production and high diversity of fishes, and this plays an important ecological role in the whole basin (Silva et al., 2007). The diminishing richness and diversity that happens from the flood season to the dry season is due to the reduction of water space (Saint-Paul et al., 2000). A greater number of species occur in larger areas, emphasizing the species-area relationship (Ricklefs, 2001). Along with this, the rise of water level - that floods the vegetation of the river banks - makes available a greater number of shelters and structurally more complex habitats as well as food resources that are not available in the dry season, supporting greater diversity in the flood season (Teixeira et al., 2005).

An important component of species diversity is evenness (Begon et al., 2007), which is the opposite of species dominance. Under natural conditions of the tropical ecosystems, rare species are distributed evenly, that is, numerous species are found with a small number of individuals. Contrary to our results, Silva et al. (2007) and Cunico et al. (2006) obtained lower evenness in the dry season, and reported the piscivores $P$. nattereri and $H$. malabaricus, respectively, as dominant species. In our research, the lower taxonomic evenness in the flood season is evidenced by the high abundance of insectivore species as $B$. alburnoides e $D$. urostriatum, which because of their feeding habits, show a strict relation with the period of high water owing to the greater availability of allochthonous insects in this period (Winemiller, 1990; Zavala-Camin, 1996; Junk, 1997; Yamamoto et al., 2004).

Assemblages can be viewed both in taxonomic or in functional terms (Poff and Allan, 1995). The use of functional groups allows us to infer more directly on the ecological responses of species to environmental variation (Hoeinghaus et al., 2006). The presence of greater diversity of trophic categories, richness of species (especially those from the top of the chain as piscivores, and basal as detritivores and herbivores) and number of trophic elements have been suggested as resulting from natural or ecological factors such 
as productivity (Giller, 1984; DeAngelis, 1992; Lowe-McConnell, 1999; Townsend et al., 1998) and stability of environments (Lowe-McConnell, 1999; Poff and Allan, 1995; Hoeinghaus et al., 2006). The type and number of trophic categories present in a community vary with the species therein, with the density of prey and with the taxonomic resolution adopted by the authors for the classification of guilds (Angermeier and Karr, 1983; Moyle and Senanayake, 1984). Studies in temperate and tropical environments reported similar results to ours, with figures ranging from five to eight guilds (Peretti and Andrian, 2004; Cassemiro et al., 2005; Rayner, 2006; Ximenes et al., 2011). Similar to the results obtained by Ximenes et al. (2011), changes in the number of trophic categories were not observed between the dry and flood seasons, suggesting that the species that were included in the community over time were grouped into the existing categories, while the available food resources in the environment are possibly exploited by both, the resident and the visitor species, and there was no variation in the number of trophic categories over time, but there were changes in their composition as changes in the water cycle allow the inclusion of new species to the community and to the categories present there. The high similarity between the trophic index values of the periods analyzed, together with the fact that these were lower than taxonomic index patterns, point to a high redundancy in the use of feeding niches by species belonging to the studied stretch in each period, indicating that, in the absence of a given species, it can be replaced by an equivalent one. Thus, our results may be related to an assembly rule proposed by Fox (1987) and Fox and Brown (1993) which is based on functional groups and that suggests that new species added to communities tend to belong to less occupied functional groups, so that a reasonably uniform species distribution among groups is achieved. We also point to evidence of the influence of seasonal changes on the qualitative and quantitative contribution of the trophic categories analyzed, possibly explained by the availability of food and food spectrum of each category.

\section{References}

ABELHA, MCF., AGOSTINHO, AA. and GOULART, E. 2001. Plasticidade trófica em peixes de água doce. Acta Scientiarum, vol. 23, no. 2, p. 425-434.

AGOSTINHO, AA. et al. 1997. Estrutura trófica. In VAZZOLER, AEAM., AGOSTINHO, AA. and HAHN, NS., orgs. A planicie de inundação do alto rio Paraná: aspectos físicos, biológicos e socioeconômicos. Maringá: Eduem. p. 229-248.
ANGERMEIER, PL. and KARR, JR. 1983. Fish communities along environmental gradients in a system of tropical streams. Environmental Biology Fishes, vol. 9, p. 117-135. http://dx.doi.org/10.1007/ BF00690857

ARRINGTON, DA. 2002. Evaluation of the relationship between habitat structure, community structure and community assembly in a Neotropical blackwater river. Texas: Texas University. [Doctor Thesis in Ecology].

BEGON, M., HARPER, JL. and TOWNSEND, CR. 2007. Ecology: individuals, populations and community. 4th ed. London: Blackwell Science. 1068 p.

BRITSKI, HA. 1999. Peixes do Pantanal: manual de identificação. Brasília: Embrapa. 64 p.

CASSEMIRO, FA., HAHN, NS. and FUGI, R. 2005. Estrutura trófica da ictiofauna, ao longo do gradiente longitudinal do reservatório de Salto Caxias (rio Iguaçu, Paraná, Brasil), no terceiro ano após o represamento. Acta Scientiarum. Biological Sciences, vol. 27 , no. 1, p. 63-71.

CASTRO, RMC. 1999. Evolução da ictiofauna de riachos sul-americanos: padróes gerais e possíveis processos causais. In CARAMASCHI, EP., MAZZONI,R., BIZERRIL, CRSF. and PERES-NETO, PR., orgs. Ecologia de Peixes de Riachos: Estado Atual e perspectivas. Rio de Janeiro: Oecologia Brasiliensis. p. 139-155.

CLARKE, KR. and GREEN, RH. 1988. Statistical design and analysis for a 'biological effects' study. Marine Ecology Progress Series, vol. 46. p. 213-26.

CLARO-JUNIOR, LH. 2003. A influência da floresta alagada na estrutura trófica de comunidades de peixes em lagos de várzea da Amazônia Central. Manaus: Instituto Nacional de Pesquisas da Amazônia. [Master Thesis in Biologia da Água Doce e Pesca Interior].

CLARO-JUNIOR, LH., FERREIRA, E., ZUANON, J. and ARAUJO-LIMA, C. 2004. O efeito da floresta alagada na alimentação de três espécies de peixes onívoros em lagos de várzea da Amazônia Central, Brasil. Acta Amazonica, vol. 34, no. 1, p. 133-137. http://dx.doi.org/10.1590/S004459672004000100018

CORREA, SB. 2005. Comparison of fish assemblages in flooded forets versus floating habitats of in upper Amazon floodplain (Pacaya Samiria National reserve, Peru). Florida: University of Florida. [Master Thesis in Ecology].

CORREA, SB. 2008. Fish assemblage structure is consistent through an annual hydrological cycle in habitats of a floodplain-lake in the Colombian Amazon. Neotropical Ichthyology, vol. 6, no. 2, p. 257-266. http://dx.doi.org/10.1590/S167962252008000200014 
COSTA, ID. 2009. Estrutura de assembléias de peixes em uma área de exploração petrolifera na Amazônia (Bacia do Rio Urucu, Amazonas, Brasil). Manaus: Instituto Nacional de Pesquisas da Amazônia. [Master Thesis in Biologia da Água Doce e Pesca Interior].

CUNICO, AM., AGOSTINHO, AA. and LATINI, JD. 2006. Influência da urbanização sobre as assembléias de peixes em três córregos de Maringá, Paraná. Revista Brasileira de Zoologia, vol. 23, no. 4, p. 1101-1110. http://dx.doi.org/10.1590/S010181752006000400018

DEPARTAMENTO NACIONAL DA PRODUÇÃO MINERAL - DNPM. 1978. Purus: geologia, geomorfologia, pedologia, vegetação e uso potencial de terra. Projeto Radambrasil. 566 p.

DeANGELIS, DL. 1992. Dynamics of nutrient cycling and food webs. Chapman and Hall. $158 \mathrm{p}$.

DORIA, CRC., VILARA, GT., ZUANON, JAS., FAVARO, LF., RIFFINO, ML. and LEITE, RG. 2005. Estudo de viabilidade das UHE's Jirau e Santo Antônio, localizadas no Rio Madeira em Rondônia, no trecho entre Porto Velho e Abunã. Rondônia: Universidade Federal de Rondônia - UNIR. 345 p. Relatório Técnico Final.

DUARTE, C. 2008. Ictiofauna associada às praias de desova de quelônios no baixo rio Purus, Amazonas, Brasil. Manaus: Instituto Nacional de Pesquisas da Amazônia. [Master Thesis in Biologia da Água Doce e Pesca Interior].

FAUTH, JE., BERNARDO, J., CAMARA, M., RESETARITS, WJJR., VAN BUSKIRK, J. and McCOLLUM, SA. 1996. Simplifying the jargon of community ecology: a conceptual approach. The American Naturalist, vol. 147, p. 282-286. http:// dx.doi.org/10.1086/285850

FERRARIS JUNIOR, C. 1991. Catfish in the aquarium. Morris Plains: Tetra Press. 199 p.

FERREIRA, EJG., ZUANON, J. and SANTOS, GM. 1998. Peixes comerciais do médio Amazonas - Região de Santarém, PA. Brasília: Imprensa Oficial. $211 \mathrm{p}$.

FERREIRA, MP. and SANTOS, ACA. 2006. Variação sazonal, recrutamento e hábitos alimentares de Tetragonopterus chalceus Spix \& Agassiz, 1829 (Characiformes, Characidae), no Rio Santo Antônio, Bahia, Brasil. Zooociências, vol. 8, no. 2, p. 117-123.

FERREIRA, CP. and CASATTI, L. 2006. Integridade biótica de um córrego na bacia do Alto Rio Paraná avaliada por meio da comunidade de peixes. Biota Neotropica, vol. 6, no. 3, p. 23-30.

FERREIRA, FC and PETRERE JRM. 2007. Anthropic effects on the fish community of Ribeirão Claro, Rio Claro, SP, Brazil. Brazilian Journal Biology, vol. 67, no. 1, p. 23-32. http://dx.doi.org/10.1590/S151969842007000100004
FOX, BJ. 1987. Species assembly and the evolution of community structure. Evolutionary Ecoogy, vol. 1, p. 201-213. http://dx.doi.org/10.1007/BF02067551

FOX, BJ and BROWN, JH. 1993. Assembly rules for functional groups in North American desert rodent communities. Oikos, vol. 67, p. 358-370. http:// dx.doi.org/10.2307/3545483

GASALLA, MA. and SOARES, LSH. 2001. Comentários sobre os estudos tróficos de peixes marinhos no processo histórico da ciência pesqueira e modelagem ecológica. Boletim do Instituto de Pesca, vol. 27, no. 2, p. 243-259.

GILLER, PS. 1984. Community Structure and the Niche. London: Chapman \& Hall. 156 p.

GODOI, DS. 2004. Diversidade e hábitos alimentares de peixes de um corrego afluente do rio Teles Pires, Carlinda, MT, drenagem do rio Tapajós. Jaboticabal: Universidade Estadual Paulista. [Master Thesis in Ecology].

GOULDING, M. 1980. The fishes and the forest: explorations in Amazonian natural history. Berkey: University of California Press. 280 p.

GOULDING, M., CARVALHO, ML. and FERREIRA, EG. 1988. Rio Negro: rich life in poor water Amazonian diversity and ecology as seen thought fish communities. California: SPB Academic Publishing. 200 p.

GURGEL, HCB., LUCAS, FD. and SOUZA, LLG. 2002. Dieta de sete espécies de peixes do semi-árido do rio Grande do Norte, Brasil. Revista Ictiología, vol. 10, no. 1-2, p. 7-16.

HAHN, NS., ALMEIDA, VLL. and LUZ, KDG. 1997. Alimentação e ciclo alimentar diário de Hoplosternum littorale (Hancock) (Siluriformes, Callichthyidae) nas lagoas Guaraná e Patos da Planície do Alto Paraná, Brasil. Revista Brasileira de Zoologia, vol. 14, no. 1, p. 57-64.

HOEINGHAUS, DJ., WINEMILLER, KO. and BIRNBAUM, JS. 2006. Local and regional determianats of stream fish assemblage structure: inference based on taxonomic vs. functional group. Journal of Biogeography, vol. 5, no. 1, p. 1-14.

JEPSEN, DB. 1997. Fish species diversity in sand bank habitats of a neotropical rivers. Environmental Biology of Fishes, vol. 49, p. 449-460. http://dx.doi. org/10.1023/A:1007371132144

JÚLIO JUNIOR, HF., CUNICO, AM., GUBIANI, EA., FERNANDES, R., DIAS, RM., GRAÇA, WJ. and GOMES, LC. 2003. Ictiofauna. UEM. Available from: <http://www.peld.uem.br/Relat2003/peldsumario03.htm\#Biótico>.

JUNK, WJ. 1993. Wetlands of Tropical South America. In WHIGHAM, D., HEJNY, S. and DYKYJOVA, D., orgs. Wetlands of the world. Boston: Dordrecht Press. 739 p. 
JUNK, WJ. 1997. General Aspects of Floodplain Ecology with Special Reference to Amazonian Floodplains. In JUNK, WJ., org. Ecological Studies. Berlin: SpringerVerlag. p. 3-20.

JUNK, W. J.; SOARES, M. G.; CARVALHO, F. M. 1983. Distribution of fish species in lake of the Amazon river floodplain near Manaus (lago camaleáo), with special reference to extreme oxygen conditions. Amazoniana, v. 7, n. 4, p. 397-431.

KUSHLAN, JA. 1976. Enviromental stability and fish community diversity. Ecology, vol. 57, no. 4, p. 821-825. http://dx.doi.org/10.2307/1936196

LAYMAN, CA. and WINEMILLER, KO. 2004. Sizebased responses of prey to piscivore exclusion in a species-rich neotropical river. Ecology, vol. 85, p. 1311-1320. http://dx.doi.org/10.1890/02-0758

LOWE-McCONNELL, RH. 1975. Fish communities in tropical freshwaters. London: Logman. $337 \mathrm{p}$.

LOWE-McCONNELL, RH. 1999. Estudos ecológicos em comunidades de peixes tropicais. São Paulo: EdUSP. 122 p.

LUDWIG, JA. and REYNOLDS, JF. 1988. Statistical ecology: a primer on methods and computing. New York: John Wiley \& Sons. 145 p.

MAGURRAN, AE. 1988. Ecological diversity and its measurement. London: Chapman \& Hall. 178 p.

MAGURRAN, AE. 2004. Measuring Biological Diversity. Oxford: Blackwell Science Ltd. 256 p.

MARINELLI, CE., FERREIRA, KM., VIEIRA, DLM., JURINITZ, CF., GUERRA, BR. and AGUIAR, AJC. 2001. Atributos ecomorfológicos para determinação de guildas tróficas da ictiofauna de igapó no arquipélago das Anavilhanas, Amazônia Central. Curso de Campo Ecologia da Floresta Amazônica. Projeto Dinâmica Biológica de Fragmentos Florestais. p. 59-62.

MÉRONA, B. and BITTENCOURT, MM. 1993. Les peuplements de poisons du "Lago do Rei", um lac d'inondation d'Amazonie centrale: description générale. Amazoniana, vol. 8, no. 3-4, p. 415-441.

MÉRONA, B. and RANKIN-DE-MÉRONA, J. 2004. Food resource partitioning in a fish community of the central Amazon floodplain. Neotropical Ichthyology, vol. 2, no. 2, p. 75-84. http://dx.doi.org/10.1590/ S1679-62252004000200004

MOURA, A. N. et al. 2010. Reservatórios do Nordeste do Brasil: biodiversidade, ecologia e manejo. Recife: Comunigraf. $540 \mathrm{p}$.

MOYLE, PB. and SENANAYAKE, FR. 1984. Resource partitioning among the fishes of rainforest streams in Sri Lanka. Journal Zoology, vol. 202, p. 195-223.

NICO, LG. and TAPHORN, DC. 1988. Food Habits of Piranhas in the Low Llanos of Venezuela.
Biotropica, vol. 20, no. 4, p. 311-321. http://dx.doi. org/10.2307/2388321

NELSON, JS. 1994. Fishes of the World. New York: John Wiley \& Sons. $600 \mathrm{p}$.

PEREIRA, RAC., RESENDE, EK. and MORAES, AS. 1996. Alimentacao de peixes detritivoros em meandros abandonados da planicie de inundacao do Rio Miranda, Pantanal, Mato Grosso do Sul, Brasil. Brasilia: Embrapa-SPI. p. 68-69.

PERETTI, D. and ANDRIAN, IF. 2004. Trophic structure of fish assemblages in five permanent lagoons of the high Parana River floodplain, Brazil. Environmental Biology Fishes, vol. 71, p. 95-103. http://dx.doi. org/10.1023/B:EBFI.0000043155.76741.a1

POFF, NL. and ALLAN, JD. 1996. Functional organization of streams fish assemblages in relation to hydrological variability. Ecology, vol. 76, no. 2, p. 606-627.

POST, DM. 2002. Using stable isotopes to estimate trophic position: models, methods, and assumptions. Ecology, vol. 83, p. 703-718. http://dx.doi. org/10.1890/0012-9658(2002)083[0703:USITE T]2.0.CO;2

POUILLY, M.; BARRERA, S.; ROSALES, C. 2006. Changes of taxonomic and trophic structure of fish assemblages along an environmental gradient in the Upper Beni watershed (Bolivia). Journal of Fish Biology, v. 68, n. 1, p. 137-156. http://dx.doi. org/10.1111/j.0022-1112.2006.00883.x

POUILLY, MF. LINO, JG., BRETENOUX, P. and ROSALES, C. 2003. Dietary-morphological relationships in a fish assemblage of the Bolivian Amazônian floodplain. Journal of Fish Biology, vol. 62, p. 1137-1158. http://dx.doi.org/10.1046/ j.1095-8649.2003.00108.x

POUILLY, M., YUNOKI, T., ROSALES, C. and TORRES, L. 2004. Trophic structure of fish assemblages from Mamoré River floodplain lakes (Bolivia). Ecology of Freshwater Fish, vol. 13, no. 4, p. 245-257. http://dx.doi.org/10.1111/j.16000633.2004.00055.x

RAYNER, TS. 2006. The trophic ecology of the freshwater fishes of an Australian rainforest river. Australia: James Cook University. [Doctor Thesis in Marine and Tropical Biology].

REIS, RE. 1997. Revision of the Neotropical catfish genus Hoplosternum (Osteriophysi: Siluriformes: Callichthyidae), with the description of two new genera and three new species. Ichthyological Exploration Freshwater, vol. 7, p. 299-326.

REIS, RE., KULLANDER, SO. and FERRAIJR, CJ. 2003. Check List of the Freshwater Fishes of South and central America. Porto Alegre: EdPUCRS. 245 p.

REZENDE, CF., CARDOSO, VT., ALBUQUERQUE, EZ. and GODOY, BS. 2005. A disponibilidade de 
recursos alimentares influencia a reprodução de peixes em florestas de igapó? Available from: <http://pdbff. inpa.gov.br/treinalp.htm>.

RICKLEFS, RE. 2001. A Economia da natureza. 5. ed. Rio de Janeiro: Guanabara Koogan. 503 p.

ROBERTSON, R. 1972. Social control of sex reversal in a coral-reef fish. Science, vol. 177, p. 9-17. http:// dx.doi.org/10.1126/science.177.4053.1007

RÖPKE, CP. 2008. Estrutura trófica das assembléias de peixes em biótopo de herbáceas aquáticas nos rios Araguaia (Tocantins) e Trombetas (Pará), Brasil. Manaus: Instituto Nacional de Pesquisas da Amazônia. [Master Thesis in Biologia da Água Doce e Pesca Interior].

SABINO, J. and ZUANON, JA. 1998. A stream fish assemblage in Central Amazonia: distribution, activity patterns and feeding behavior. Ichthyological Exploration of Freshwaters, vol. 8, p. 201-210.

SAINT-PAUL, U., ZUANON, JAS., CORREA, MAV., GARCIA, M., FABRÉ, NN., BERGER, U. and JUNK, WJ. 2000. Fish communities in central Amazônian white and blackwater floodplains. Environmental Biology of Fishes, vol. 57, p. 235-250. http://dx.doi.org/10.1023/A:1007699130333

SANTOS, CL.; SANTOS, IA. and SILVA, CJ. 2009. Ecologia trófica de peixes ocorrentes em bancos de macrófitas aquáticas na baia Caiçara, Pantanal Mato-Grossense. Revista Brasileira de Biociências, vol. 7, p. 473-476.

SANTOS, GM. 1981. Estudos de alimentação e hábitos alimentares de Schizodon fasciatus Agassiz, 1829, Rhytiodus microlepis Kner, 1859 e Rhytiodus argentofuscus Kner, 1859, do lago Janauacá - AM. Acta Amazonica, vol. 11, p. 267-283.

SANTOS, GM. 1991. Pesca e Ecologia dos Peixes de Rondônia. Manaus: Instituto Nacional de Pesquisas da Amazônia. [Doctor Thesis in Biologia da Água Doce e Pesca Interior].

SANTOS, GM., FERREIRA, EJG. and ZUANON, JAS. 2006. Peixes Comerciais de Manaus. Manaus: ProVárzea. $144 \mathrm{p}$.

SANTOS, GM., MERONA, B., JURAS, AA. and JEGU, M. 2004. Peixes do baixo rio Tocantins: 20 anos depois da usina hidrelétrica Tucuruí. Brasília: Eletronorte. 215 p.

SANTOS, GM. and ROSA, PS. 1998. Alimentação de Anostomus ternetzi e Synaptolaemus cingulatus, duas espécies de peixes amazônicos com boca superior. Revista Brasileira de Biologia, vol. 58, p. 255-262.

SANTOS JUNIOR, UM. 2003. Ecofisiologia de espécies arbóreas plantadas sobre área degradada por atividade petrolifera na Amazônia Central. Manaus: Instituto Nacional de Pesquisas da Amazônia. [Master Thesis in Biologia da Água Doce e Pesca Interior].
SILVA, EF., MELO, CE. and VENERE, PC. 2007. Fatores que influenciam a comunidade de peixes em dois ambientes no Rio das Mortes, Planície o Bananal, Mato Grosso, Brasil. Revista Brasileira de Zoologia, vol. 24, no. 2, p. 482-492. http://dx.doi. org/10.1590/S0101-81752007000200029

SILVA, FFG. 2008. Composição e distribuição da ictiofauna do Rio Guaraguaçu (Paranaguá, ParanáBR) e biologia alimentar de três espécies. Curitiba: Universidade Federal do Paraná, Curitiba. [Master Thesis in Ecology].

SIQUEIRA-SOUZA, FK. and FREITAS, CEC. 2004. Fish diversity of floodplain lakes on the lower stretch of the Solimóes river. Brazillian Journal Biology, vol. 64, p. 501-10.

SOUSA, RGC. and FREITAS, CEC. 2008. The influence of flood pulse on fish communities of floodplain canals in the Middle Solimóes River, Brazil. Neotropical Ichthyology, vol. 6, no. 2 p. 249-255.

TEIXEIRA, TP., PINTO, BCT., TERRA, BF., ESTILIANO, EO., GRACIA, D. and ARAÚJO, FG. 2005. Diversidade das assembléias de peixes nas quatro unidades geográficas do rio Paraíba do Sul. Iheringia, Série Zoologia, vol. 95, no. 4, p. 347-357.

THOMAZ, SM., ROBERTO, MC. and BINI, LM. 1997. Caracterização limnológica dos ambientes aquáticos e influência dos níveis fluviométricos. In VAZZOLER, AEAM., AGOSTINHO, AA. and HAHN, NS., eds. A Planície de Inundação do Alto Rio Paraná: aspectos físicos, biológicos e socioeconômicos. Maringá: EdUEM - Nupelia. p. 73-102.

TOWNSEND, CR., THOMPSON, RM., MCINTOSH, AR., KILROY, C., EDWARDS, E. and SCARSBROOK, MR. 1998. Disturbance, resource supply, and food-web architecture in streams. Ecology Letters, vol. 1, no. 3, p. 200-209.

VALE, JD. 2003. Composição, diversidade e abundância da ictiofauna na área do Catalão, Amazônia Central. Manaus: Instituto Nacional de Pesquisas da Amazônia. [Master Thesis in Biologia da Água Doce e Pesca Interior].

VIEIRA, LS. and SANTOS, PCTC. 1987. Amazônia: Seus solos e outros recursos naturais. São Paulo: Agronômica Ceres. 416 p.

XIMENES, LQL., MATEUS, LAF. and PENHA, JMF. 2011. Variação temporal e espacial na composição de guildas alimentares da ictiofauna em lagoas marginais do Rio Cuiabá, Pantanal Norte. Biota Neotropica, vol. 11, no. 1, p. 205-215. http:// dx.doi.org/10.1590/S1676-06032011000100022

WINEMILLER, KO. 1990. Spatial and temporal variation in tropical fish trophic networks. Ecological Monographs, v. 60, p. 331-367. http://dx.doi. org/10.2307/1943061 
WINEMILLER, KO. 2004. Floodplain river food webs: generalizations vand implications for fisheries management. In WELCOMME, RL. and PETR, T., orgs. Proceedings of the Second International Symposium on the Management of Large Rivers for Fisheries. Bangkok: RAP Publication, Food and Agriculture Organization of the United Nations \& Mekong River Commission. 310 p.

WINEMILLER, KO. and JEPSEN, D.B. 1998. Effects of seasonality and fish movement on tropical river food webs. Journal of fish Biology, vol. 53, p. 267-
296. http://dx.doi.org/10.1111/j.1095-8649.1998. tb01032.x

WOLDA, H. 1981. Similarity indices, sample size and diversity. Oecologia, vol. 50, p. 296-302. http:// dx.doi.org/10.1007/BF00344966

YAMAMOTO, KC., SOARES, MGM. and FREITAS, CEC. 2004. Alimentação de Triportheus angulatus (Spix \& Agassiz, 1829) no lago Camaleão, Manaus, AM, Brasil. Acta Amazonica, vol. 34, p. 653-659.

ZAVALA-CAMIN, LA. 1996. Introdução aos estudos sobre alimentação natural de peixes. Maringá: EdUEM. 129 p.

Received: 11 January 2013 Accepted: 03 July 2013 